\title{
Soluble epoxide hydrolase derived lipid mediators are elevated in bronchoalveolar lavage fluid from patients with sarcoidosis: a cross-sectional study
}

Marcus O. D. Sjödin ${ }^{1,2 \dagger}$, Antonio Checa ${ }^{1 \dagger}$, Mingxing Yang ${ }^{3}$, Sven-Erik Dahlén ${ }^{2}$, Åsa M. Wheelock ${ }^{3}$, Anders Eklund $^{3}$, Johan Grunewald ${ }^{3+}$ and Craig E. Wheelock ${ }^{1 *+}$ (D)

\begin{abstract}
Background: Sarcoidosis is a systemic inflammatory multi-organ disease almost always affecting the lungs. The etiology remains unknown, but the hallmark of sarcoidosis is formation of non-caseating epithelioid cells granulomas in involved organs. In Scandinavia, > 30\% of sarcoidosis patients have Löfgren's syndrome (LS), an acute disease onset mostly indicating a favorable prognosis. The impact of dysregulation of lipid mediators, which has been investigated in other inflammatory disorders, is still unknown.

Methods: Using three different liquid chromatography coupled to tandem mass spectrometry targeted platforms (LC-MS/MS), we quantified a broad suite of lipid mediators including eicosanoids, sphingolipids and endocannabinoids in bronchoalveolar lavage (BAL) fluid from pulmonary sarcoidosis patients $(n=41)$ and healthy controls $(n=16)$.

Results: A total of 47 lipid mediators were consistently detected in BAL fluid of patients and controls. After false discovery rate adjustment, two products of the soluble epoxide hydrolase ( $\mathrm{SEH}$ ) enzyme, 11,12-dihydroxyeicosa5,8,14-trienoic acid (11,12-DiHETrE, $p=4.4 \mathrm{E}-5, \mathrm{q}=1.2 \mathrm{E}-3$, median fold change $=6.0)$ and its regioisomer 14,15dihydroxyeicosa-5,8,11-trienoic acid (14,15-DiHETrE, $p=3.6 \mathrm{E}-3, q=3.2 \mathrm{E}-2$, median fold change $=1.8$ ) increased in patients with sarcoidosis. Additional shifts were observed in sphingolipid metabolism, with a significant increase in palmitic acid-derived sphingomyelin (SM16:0, $p=1.3 \mathrm{E}-3, \mathrm{q}=1.7 \mathrm{E}-2$, median fold change $=1.3$ ). No associations were found between these 3 lipid mediators and LS, whereas levels of SM 16:0 and 11,12-DiHETrE associated with radiological stage $(p<0.05)$, and levels of 14,15 -DiHETrE were associated with the BAL fluid CD4/CD8 ratio.

Conclusions: These observed shifts in lipid mediators provide new insights into the pathobiology of sarcoidosis and in particular highlight the sEH pathway to be dysregulated in disease.
\end{abstract}

Keywords: Sarcoidosis, Eicosanoids, Sphingolipids, Endocannabinoids, Mass spectrometry, Inflammation

\footnotetext{
* Correspondence: Craig.Wheelock@ki.se; craig.wheelock@ki.se

${ }^{\dagger}$ Marcus O. D. Sjödin, Antonio Checa, Johan Grunewald and Craig E.

Wheelock contributed equally to this work.

'Division of Physiological Chemistry II, Department of Medical Biochemistry \&

Biophysics, Karolinska Institutet, 17177 Stockholm, Sweden

Full list of author information is available at the end of the article
}

C The Author(s). 2018 Open Access This article is distributed under the terms of the Creative Commons Attribution 4.0 International License (http://creativecommons.org/licenses/by/4.0/), which permits unrestricted use, distribution, and reproduction in any medium, provided you give appropriate credit to the original author(s) and the source, provide a link to the Creative Commons license, and indicate if changes were made. The Creative Commons Public Domain Dedication waiver (http://creativecommons.org/publicdomain/zero/1.0/) applies to the data made available in this article, unless otherwise stated. 


\section{Background}

Sarcoidosis is a systemic granulomatous disease of unknown etiology [1]. The primary impacted organ are the lungs in $>90 \%$ of the patients, but virtually any organ can be affected [2]. In Sweden, a prevalence of 160 cases per 100,000 individuals has been estimated [3]. In $20-40 \%$ of the cases the disease may start suddenly with an acute manifestation known as Löfgren's syndrome (LS), with high fever, erythema nodosum and/or bilateral ankle arthritis, and bilateral hilar lymphadenopathy $[2,4-6]$. However, the majority will have an insidious onset with fatigue, dry cough, dyspnea, low-grade fever, chest pain and sometimes weight loss (non-LS). The pathogenesis is still unknown, but some evidence indicates that causative antigen(s) enter the host and are phagocytosed by antigen-presenting cells (APCs), predominantly macrophages or dendritic cells. The APCs subsequently present the antigen via human leukocyte antigen (HLA) class II molecules primarily to a restricted set of $\mathrm{CD}^{+}{ }^{+} \mathrm{T}$ lymphocytes [7]. The development of sarcoidosis seems to involve the intricate balance in the trimolecular complex consisting of an antigen, HLA class II molecules and T-cell receptors [8]. A genetic influence is evident as increased familial occurrence as well different disease modes in different ethnic groups have been shown [9]. The strongest genetic association is found within the HLA alleles that clearly associate with disease risk and phenotype [10]. For example, patients with the HLA-DRB1*0301 allele are known to associate with LS and a good prognosis with complete resolution within 2 years without treatment [10-12].

In the absence of a causative agent, sarcoidosis remains a diagnosis of exclusion of other diseases. There are no definitive diagnostic blood or radiologic imaging tests specific for the disorder, but there are several tests that aid in diagnosis [4], such as increased levels of serum ACE [13], chitotriosidase [14] or sIL-2R [15]. A cell pattern typical of lymphocytic alveolitis and a $\mathrm{T}$-cell $\mathrm{CD}^{+} / \mathrm{CD}^{+}$ratio $>3.5$ have also been recognized [16]. However, no single biomarker of sarcoidosis has been reported that has sufficient specificity, sensitivity and reproducibility to reliably diagnose the disease [16]. Given the need for a molecular signature of the disease, this study was designed to investigate potential dysregulations of lipid mediators in patients with sarcoidosis. Lipid mediators are biologically active molecules that possess multiple physiological roles including cell structure, innate immune defense, homeostasis and in particular play a distinct role in inflammatory processes [17]. The role of lipid mediators in the etiology and pathology of respiratory diseases has been demonstrated, including asthma [18, 19], COPD [20] and cystic fibrosis [21]. Accordingly, in this study, three lipid mediator classes, eicosanoids, endocannabinoids and sphingolipids, were quantified in bronchoalveolar lavage (BAL) fluid from sarcoidosis patients and healthy controls. To our knowledge, the current study is the first to perform metabolic profiling of lipid mediators in newly diagnosed sarcoidosis.

\section{Methods \\ Study population, bronchoscopy and bronchoalveolar lavage}

This cross-sectional study consisted of 41 patients with sarcoidosis and 16 healthy volunteers as controls. Due to its influence upon lipid mediator levels [22], smoking was set as an exclusion criterion. All patients were referred to the Respiratory Medicine Unit at the Karolinska University Hospital (Stockholm, Sweden) on the suspicion of sarcoidosis for diagnostic investigations, including bronchoscopy and the morning collection of bronchoalveolar lavage (BAL) fluid as previously described [23]. The investigations were performed as close to onset of symptoms as possible and as soon as sarcoidosis was suspected. All patients were HLA-typed (Additional file 1) and diagnosed with sarcoidosis through typical clinical and radiographic manifestations, findings at bronchoscopy with biopsies revealing granuloma (not required for LS) and/or BAL fluid including an elevated CD4/CD8-ratio (> 3.5). Diagnoses were in accordance with the criteria established by the World Association of Sarcoidosis and other Granulomatous Disorders (WASOG) [24]. Chest radiography was classified as stage I (bilateral hilar lymphadenopathy, BHL) and stage II (BHL with parenchymal infiltrates). Lung function was analyzed with regard to $\mathrm{FEV}_{1}$ and FVC. All patients had symptoms compatible with an active disease, two of them were on prednisolone treatment at the time of BAL sampling, while four patients were on treatment with local steroids. Only two patients declared use of non-steroid anti-inflammatory drugs (NSAID) at the time of sampling. Healthy controls were recruited by announcement on a website used for recruiting healthy volunteers at the Karolinska University Hospital. A clinical examination was performed and blood was drawn for analyses of CRP, blood status and electrolytes. All volunteers had a normal chest $\mathrm{X}$-ray at least 3 weeks prior to bronchoscopy and none showed signs of infection. Written informed consent was obtained from all subjects. None of the subjects had signs of any airway infection or allergy symptoms at the time of BAL sampling. Subjects diagnosed with asthma, COPD, and other lung diseases, or other inflammatory conditions were excluded from the study. The Regional Ethical Review Board in Stockholm approved the study. Samples were aliquoted and stored at $-80^{\circ} \mathrm{C}$ until analysis. On the day of extraction, after thawing at $4{ }^{\circ} \mathrm{C}\left(\mathrm{n}_{\max }=24\right)$, samples were aliquoted for independent extractions of eicosanoids and endocannabinoids (Section 2.3.1) and sphingolipids (Section 2.3.2). 


\section{Chemical standards}

Eicosanoids and endocannabinoids were purchased from Cayman Chemical (Ann Arbor, USA), with the exception of 12(13)-EpODE, 9,10,13-TriHOME and 9,12,13-TriHOME from Larodan (Solna, Sweden) and $\mathrm{LTB}_{5}$ from Enzo (Solna, Sweden). Sphingolipids were purchased from Avanti Polar Lipids (Alabaster, USA). A complete list of lipid mediator nomenclature is provided in Additional file 2. Methanol, acetonitrile, isopropanol, acetic and formic acid were of LC-MS grade from Fischer Scientific (Stockholm, Sweden). Ethyl acetate and chloroform were purchased from Sigma-Aldrich (St. Louis, USA).

\section{Sample extraction}

For eicosanoids and endocannabinoids, a volume of 3 $\mathrm{mL}(n=53)$ and a minimum volume of $2.5 \mathrm{~mL}(n=4)$ of BAL fluid was spiked with $10 \mu \mathrm{L}$ of isotopically labeled internal eicosanoid and endocannabinoid standards (Additional file 3) and extracted with HLB Oasis solid phase extraction columns $(3 \mathrm{cc} / 60 \mathrm{mg}$, Waters) as previously described [22]. Detailed information on the extraction is provided in Additional file 4.

For sphingolipids, a volume of $200 \mu \mathrm{L}$ of BAL fluid in Eppendorf tubes were spiked with $10 \mu \mathrm{L}$ of sphingolipid internal standards (Additional file 3) and subjected to a modified Bligh \& Dyer extraction as previously described [25]. Detailed information on the extraction is provided in the Additional file 4.

\section{LC-MS/MS analysis}

Eicosanoids, endocannabinoids and sphingolipids were screened using 3 separate targeted liquid chromatography tandem mass spectrometry (LC-MS/MS) lipid mediator platforms. Chromatographic separation was performed on an Acquity $^{\text {mix }}$ UPLC system from Waters (Milford, MA, USA). Detection was carried out on a Xevo TQ-S (Waters) mass spectrometer with electrospray ionization operating in selected reaction monitoring (MRM) mode. Detailed information of the separation and general MS conditions is provided in Additional file 5. Retention times and SRM transitions for each compound can be found elsewhere $[22,25,26]$.

\section{Statistical analysis}

Values below the lowest limit of quantification (LLOQ) were replaced by the $25 \%$ of the lowest detected concentration for that compound (Additional file 1). Univariate statistical analyses were performed using $\mathrm{R}$ (version 3.4.0). Fold changes were calculated by using the median between groups. A two-tailed Mann-Whitney U-test was used for comparisons between two groups. Multiple hypothesis testing was controlled according to Storey (q-value) using the QVALUE software [27]. A q-value lower than 5\% was considered statistically significant.
Correlation analyses were performed using Spearman's correlation (two-tailed). Trends in the shifts of lipid mediators with chest radiography stages were evaluated using the Cochran-Armitage trend test for linearity. The median value among the healthy controls for each lipid mediator was used as a cut-off limit for all three groups and all trend-based $p$-values were one-tailed.

\section{Results \\ Demographics}

Cohort demographics are summarized in Table 1. A total of 21 subjects had LS and the patients presented with lower FEV ${ }_{1}$ and FVC scores relative to controls. No differences were observed in lung function between LS and non-LS patients or with regard to radiographic stage.

\section{Lipid mediators in BAL fluid}

A total of 47 lipids (eicosanoids: $n=26$, sphingolipids: $n=17$ and endocannabinoids: $n=4$ ) were consistently detected in the samples and are summarized in Additional file 6 .

\section{Differences between controls and patients with sarcoidosis}

A total of 2 eicosanoids differed $(\mathrm{q}<0.05)$ between controls and patients with sarcoidosis (Fig. 1 a, b, Additional file 6). The arachidonic acid-derived (Fig. 1f) 11,12-DiHETrE $\left(p=4.4 \times 10^{-5}, \mathrm{q}=1.2 \times 10^{-3}\right)$ and its regioisomer $14,15-\operatorname{DiHETrE} \quad(p=3.6 \times 10-3, \quad \mathrm{q}=3.1 \times$ $10-2)$ were increased (6.0- and 1.8-fold median change, respectively) in patients with sarcoidosis relative to controls. Additionally, three linoleic acid-derived eicosanoids, 13-HODE $\left(p=3.3 \times 10^{-2}, \mathrm{q}=1.2 \times 10^{-1}, \quad \mathrm{FC}=\right.$ 1.6), 9(10)-EpOME $\left(p=3.9 \times 10^{-2}, \mathrm{q}=1.2 \times 10^{-1}, \mathrm{FC}=\right.$ $0.6)$ and 12(13)-EpOME $\left(p=4.5 \times 10^{-2}, \mathrm{q}=1.2 \times 10^{-1}\right.$, $\mathrm{FC}=0.7)$ were dysregulated in patients with sarcoidosis relative to controls $(p<0.05)$, but were above the FDR threshold (Fig. 1 c-e).

One sphingolipid (SM 16:0 $\left[p=1.3 \times 10^{-3}, \mathrm{q}=1.7 \times\right.$ $\left.10^{-2}, \mathrm{FC}=1.3\right]$ was found increased in patients with sarcoidosis relative to controls. Additionally two sphingomyelins (SM 18:0; $p=2.7 \times 10^{-2}, \mathrm{q}=1.3 \times 10^{-1}, \mathrm{FC}=1.2$ and SM 24:1; $\left.\mathrm{p}=3.6 \times 10^{-2}, \mathrm{q}=1.3 \times 10^{-1}, \quad \mathrm{FC}=1.5\right)$, and one hexosylceramide (HexCer 16:0; $p=1.6 \times 10^{-2}$, $\mathrm{q}$ $\left.=1.0 \times 10^{-1}, \mathrm{FC}=1.6\right)($ Fig. 2), were elevated in patients with sarcoidosis relative to controls $(p<0.05)$, but were above the FDR threshold.

There were no differences $(\mathrm{p}<0.05)$ between patients with sarcoidosis and healthy controls in endocannabinoid levels (Additional file 6). None of the compounds that were dysregulated between the groups correlated with age in either the healthy or sarcoidosis groups (Additional file 7). 
Table 1 Patient demographics from healthy controls and patients with sarcoidosis included in the current study. Demographics of patients with sarcoidosis according to the presence or absence of Löfgren's syndrome and X-ray stage are also presented

\begin{tabular}{|c|c|c|c|c|c|c|}
\hline & Healthy control & $\begin{array}{l}\text { Sarcoidosis } \\
\text { (All patients) }\end{array}$ & Sarcoidosis non-Löfgren & Sarcoidosis Löfgren & $\begin{array}{l}\text { Sarcoidosis } \\
\text { X-ray stage I }\end{array}$ & $\begin{array}{l}\text { Sarcoidosis } \\
\text { X-ray stage II }\end{array}$ \\
\hline Number & 16 & 41 & 20 & 21 & 22 & 19 \\
\hline Age, years & 23 [23-25] & $40[33-47]^{e}$ & $43[33-52]$ & 40 [35-43] & $41[36-48]$ & 40 [32-46] \\
\hline Sex, female/male & $8 / 8$ & $15 / 26$ & $7 / 13$ & $8 / 13$ & $7 / 15$ & $8 / 11$ \\
\hline CD4/CD8 & $1.8[1.4-2.7]^{\mathrm{a}}$ & $8.7[4.8-11.5]^{\mathrm{e}}$ & $7.0[4.3-11.0]$ & $9.6[4.9-13.0]$ & $9.7[5.0-12.6]$ & $7.3[3.8-11.3]$ \\
\hline $\mathrm{X}$-ray stage, $1 / \| 1$ & - & $22 / 19$ & $8 / 12$ & $14 / 7$ & $22 / 0$ & 0/19 \\
\hline$\% F V_{1}$ & 111 [101-118] & $89[81-100]^{e}$ & 89 [80-101] & 89 [82-100] & 88 [81-100] & 89 [82-101] \\
\hline$\% F V C$ & 114 [109-117] & $90[81-99]^{\mathrm{b}, \mathrm{e}}$ & $91[79-101]^{c}$ & $90[82-96]^{d}$ & $90[81-97]^{\mathrm{e}}$ & $91[81-100]^{e}$ \\
\hline
\end{tabular}

Age, CD4/CD8, \%FEV 1 and \%FVC are presented as median [Interquartile range]

${ }^{a} n=13$

${ }^{b} n=28$

${ }^{c} n=16$

${ }^{d} n=12$

e $p<0.05,2$ tailed t-test

\section{Lipid mediator associations with signs of disease staging} and activity

For lipid mediators differing between controls and patients with sarcoidosis after false discovery rate adjustment $(\mathrm{q}<$ 0.05), associations with LS, CD4/CD8 ratio, chest radiograph stage, $\% \mathrm{FEV}_{1}$ and $\% \mathrm{FVC}$ were performed (Figs. 3 and 4). Due to the exploratory nature of this study, associations of lipid mediators with $p<0.05$ were also performed and are presented in Additional file 8. For these compounds, a weak correlation was found between levels of 14,15-DiHE$\operatorname{TrE}\left(\mathrm{r}_{\mathrm{s}}=0.33, p=3.4 \times 10 \mathrm{E}^{-2}\right)$ and $\mathrm{CD} 4 / \mathrm{CD} 8$ ratio (Fig. $\left.3 \mathrm{~b}\right)$. No differences were found between patients with or without Löfgren syndrome (Fig. 3d, e). Levels of 11,12-DiHETrE $\left(p=2.2 \times 10^{-4}\right)$ and SM 16:0 $\left(p=2.5 \times 10^{-2}\right)$ increased with radiography stage according to the Cochran-Armitage test (Fig. $3 g$ and i). Weak associations with \%FVC were found

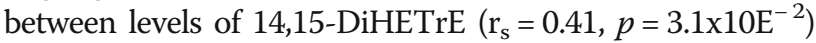
and SM 16:0 $\left(\mathrm{r}_{\mathrm{s}}=0.50, p=7.0 \times 10 \mathrm{E}^{-3}\right)($ Fig. 4).

\section{Discussion}

Given the known role of lipid mediators in inflammation $[18,19]$, and specifically pro-inflammatory eicosanoids and sphingolipids [17], in respiratory disease, this exploratory study was designed to profile these lipid mediators in BAL fluid of patients with sarcoidosis. We hypothesized that the findings would identify molecular descriptors of the disease. In addition, it was expected that increased knowledge of the dynamics surrounding lipid mediator production in sarcoidosis would provide improved understanding of disease processes in the organ most commonly affected by the disease.

Multiple lipid mediators were quantified in the BAL fluid, further supporting that this material is useful for monitoring biochemical process in the lung. The primary observed shifts in lipid mediators were increases in the BAL fluid concentrations of 11,12-DiHETrE and
14,15-DiHETrE with sarcoidosis (Fig. 1). Interestingly, the concentration of 11,12-DiHETrE also increased with radiography stages $\mathrm{I}$ and II in patients with sarcoidosis. These compounds are formed via the cytochrome P450-mediated oxidation of arachidonic acid to the corresponding epoxyeicosatrienoic acids (EpETrEs): 11(12)-EpETrE and 14(15)-EpETrE [28]. Out of the 4 regioisomers of epoxyeicosatrienoic acids screened (Fig. 1f), only 11(12)-EpETrE was observed, but this compound was not altered in patients. The EpETrEs can be metabolized via multiple pathways [29], with the soluble epoxide hydrolase ( $\mathrm{sEH}$ ) being the dominant pathway in most tissues [28]. The EpETrEs are generally considered to exert anti-inflammatory properties, while the DiHETrEs are pro-inflammatory [30, 31]. These opposing effects have been observed in models of pulmonary diseases [32]. A combination of sEH inhibitors and EpETrE treatment reduced tobacco smoke-induced inflammation, with an concomitant decrease in DiHETrE in a smoking rat model, demonstrating that sEH inhibition can attenuate at least part of the acute inflammation associated with tobacco exposure [32]. Accordingly, the observed increase in DiHETrEs suggests an inflammatory response, which is also in agreement with other reported markers of an ongoing inflammation in sarcoidosis [16]. The $\mathrm{sEH}$ pathway has also been suggested as a potential therapeutic target in cystic fibrosis [21], COPD [22] and asthma [33]. Clinical trials are currently underway to investigate how cytochrome P450 epoxygenase pathway enzymes, including the $\mathrm{sEH}$, affect macrophage function in the lungs and inflammatory responses (clinicaltrials.gov NCT02743468) as well as in COPD (clinicaltrials.gov NCT01762774). Interestingly, the linoleic acid-derived 9(10)- and 12(13)-EpOME (structural analogs of the arachidonic acid-derived EpETrEs) were decreased in patients with 


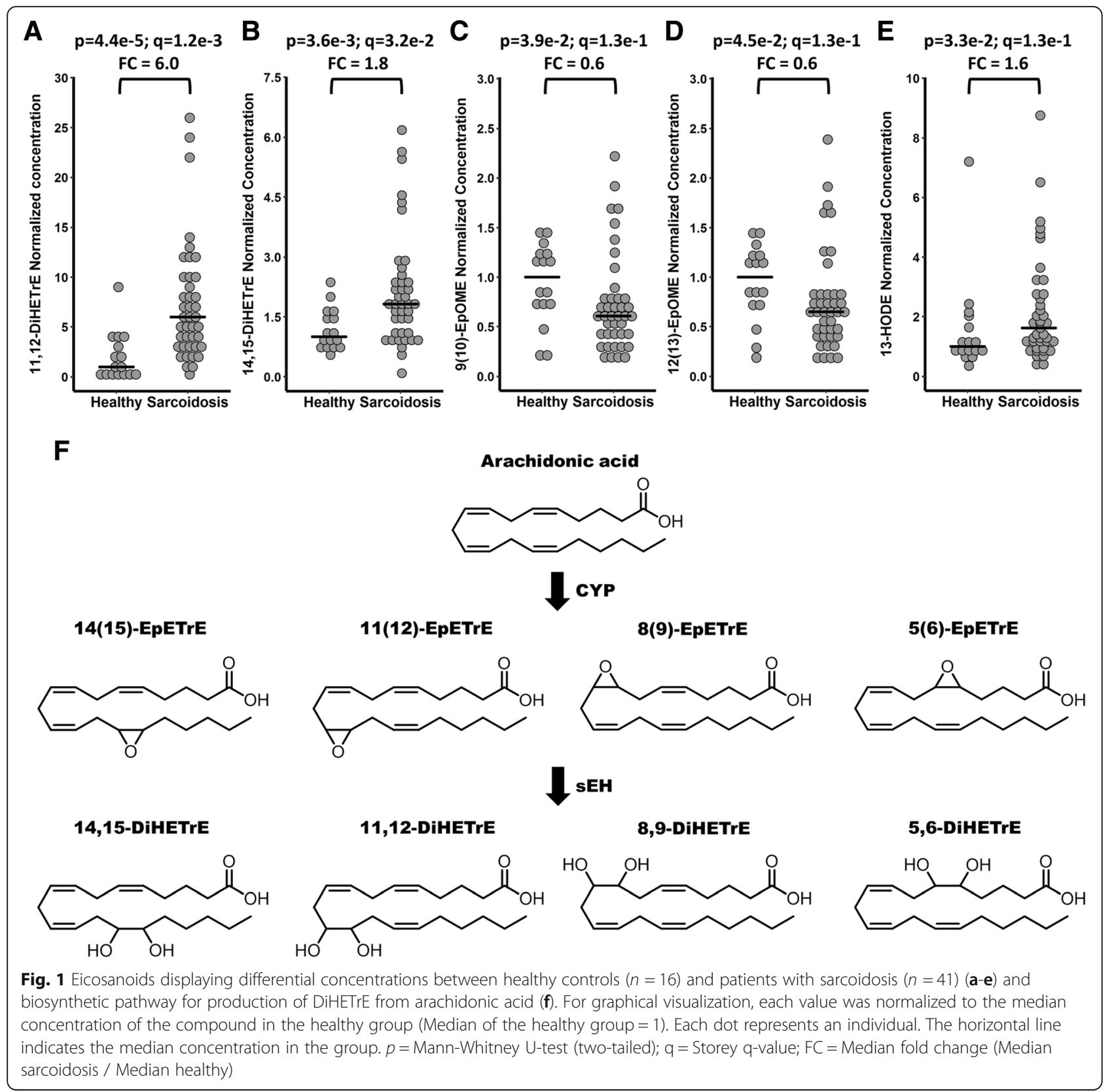

sarcoidosis. Though these compounds are also metabolized via the sEH to form the corresponding vicinal diols, no differences were found between patients and controls in the BAL fluid levels of the downstream products (9,10-DiHOME and 12,13-DiHOME, respectively). These data suggest that the lipid mediator dysregulations in sarcoidosis are specific for the arachidonic acid pathway, as opposed to COPD [22], which demonstrates dysregulation in the linoleic acid-derived mediators.

Changes in sphingolipid metabolism involved multiple lipid species, including SM (16:0, 18:0, 24:1) and HexCer (16:0). Sphingomyelins have been previously reported to increase in the BAL fluid of sarcoidosis patients [34]. These sphingolipids are biochemically linked, with ceramides acting as the intermediate for the formation of both sphingomyelins and hexosylceramides (Fig. 2e). Sphingolipids have an essential role as structural components of membranes, but also serve as critical signaling molecules [35]. They are also involved in the pathophysiology of many diseases including microbial infections [36], Alzheimer's disease [37] and respiratory disease [38]. For example, in asthma the ORMDL genes have been identified as a risk factor for childhood asthma [39]. Moreover several biosynthetic intermediates in the sphingolipid biosynthesis, including 


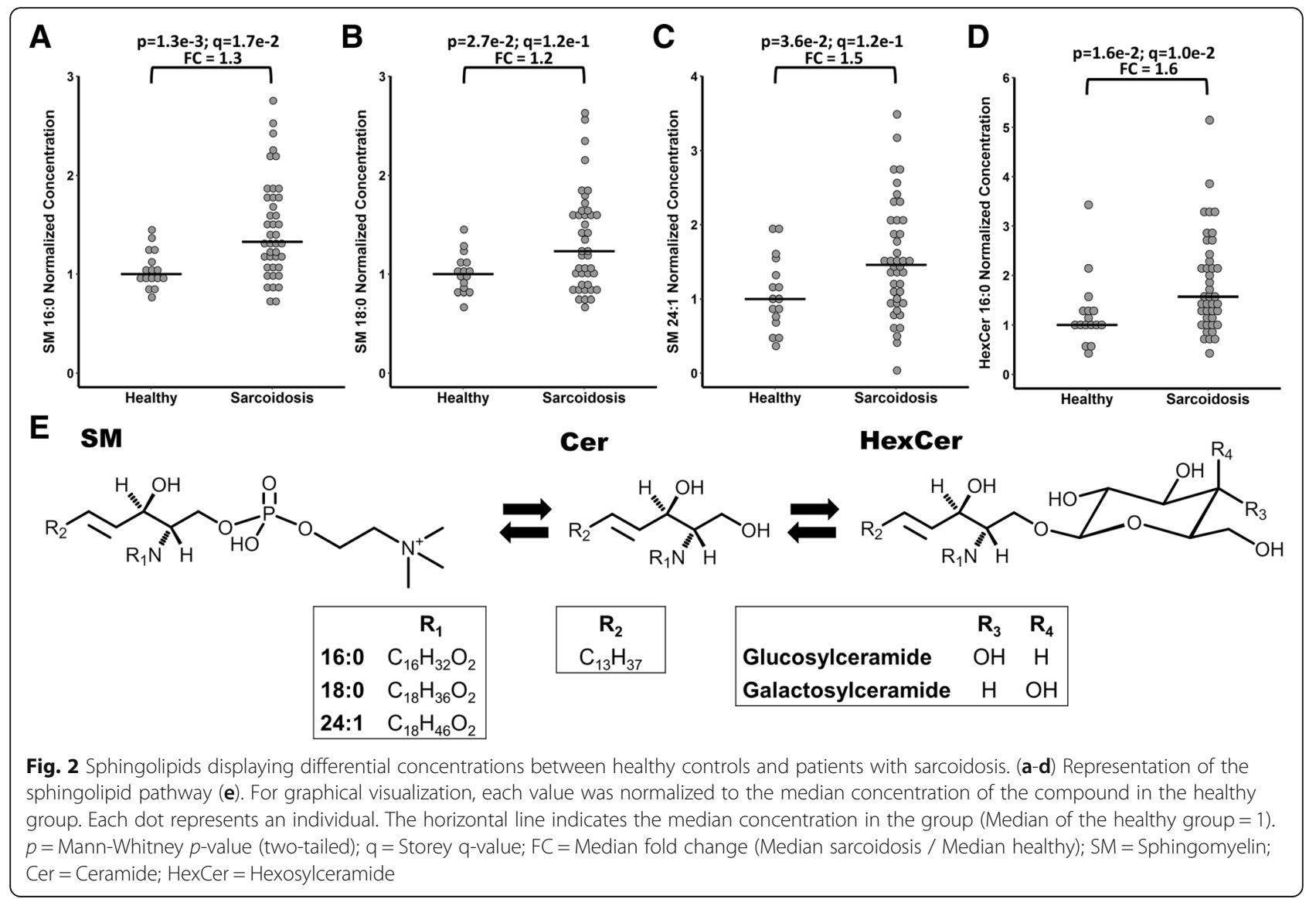

ceramides and sphingosine-1-phosphate have been shown to affect immune-cell trafficking, mast cell degranulation and airway hyper-responsiveness in asthma-associated inflammatory processes [40-42]. In the current study, a weak association between SM 16:0 and radiography stage was observed. Further studies should include patients with advanced disease stages to more closely investigate possible associations of sphingomyelins with disease development.

There are some limitations in this study. First, there is an age imbalance between sarcoidosis patients and healthy controls. However, none of the dysregulated metabolites was associated with age in patients or controls. Two patients were on prednisolone treatment and four more were on local steroids at the time of sampling, but they did not exhibit different profiles in the reported lipid mediators (data not shown), which does not suggest a strong effect of steroid treatment upon lipid mediator profiles. Additionally, patients with more advanced stages of the disease would be needed in order to confirm the association of the identified markers with the sarcoidosis radiological stage. On the other hand, this study explored the lipid mediator profiles close to disease onset, minimizing any potential effects associated with ongoing treatment, and highlighting disease-specific processes. In addition, the specificity of the observed dysregulations in lipid mediator levels need to be established relative to other respiratory diseases. Lastly, an obstacle to working with BAL fluid is the lack of clear protocols to perform data normalization. Previous attempts have been made to normalize the data using for example recovery volume [43] or metabolite markers [44], but there are of as yet no commonly accepted protocols.

\section{Conclusions}

This is the first study to profile lipid mediators in sarcoidosis, providing useful information on the levels of these potent biological mediators in this disease. The findings suggest that arachidonic acid-derived products of the $\mathrm{sEH}$ pathway as well as some sphingolipid species (particularly SM 16:0) may serve as molecular descriptors of sarcoidosis. Of particular interest are the observations that the primary altered pathways are similar to those observed in other respiratory diseases, including asthma, COPD, and cystic fibrosis, suggesting that the diseases share a similar inflammatory component. The predominant shifts were in $\mathrm{sEH}$ products of arachidonic acid metabolism, further 


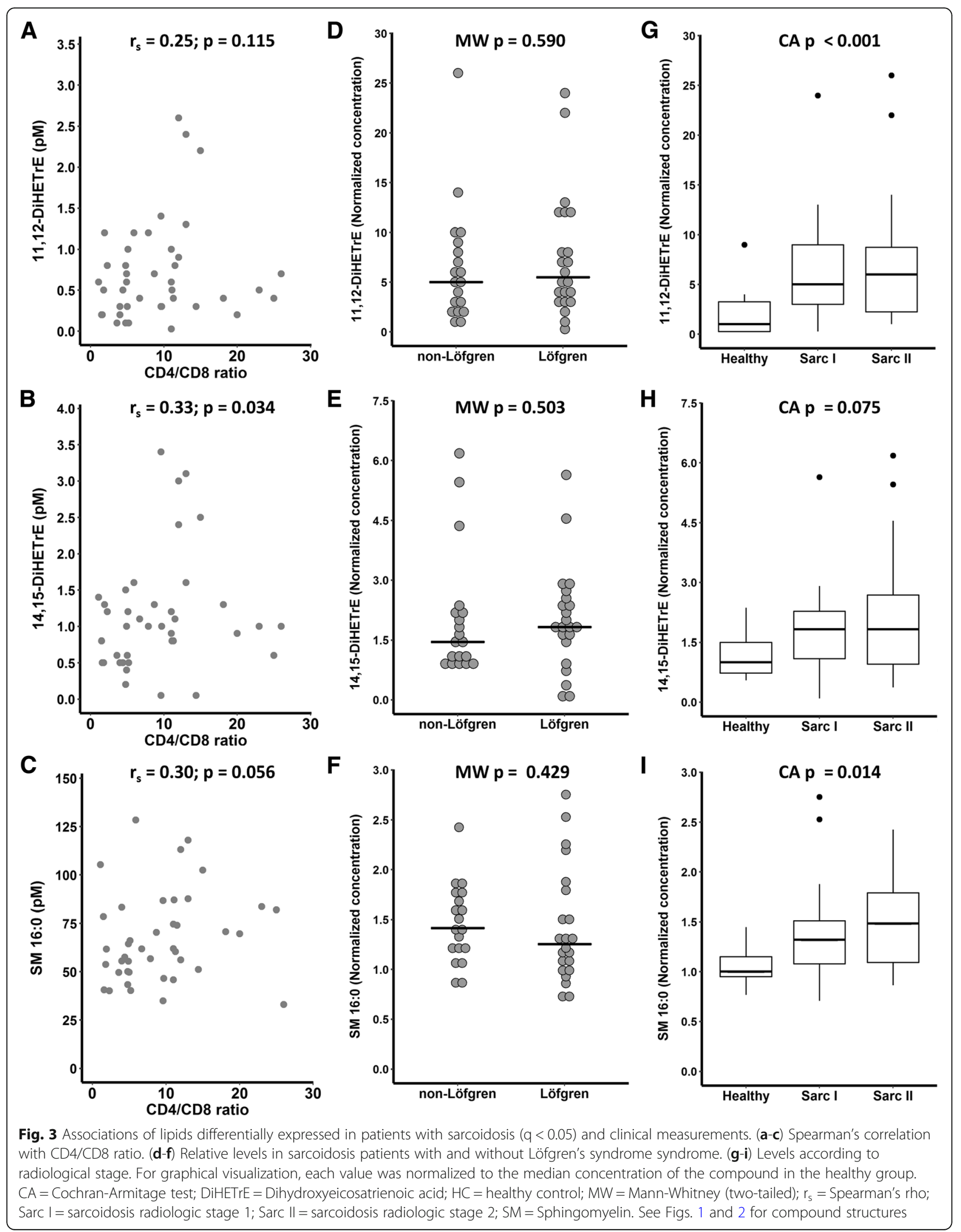



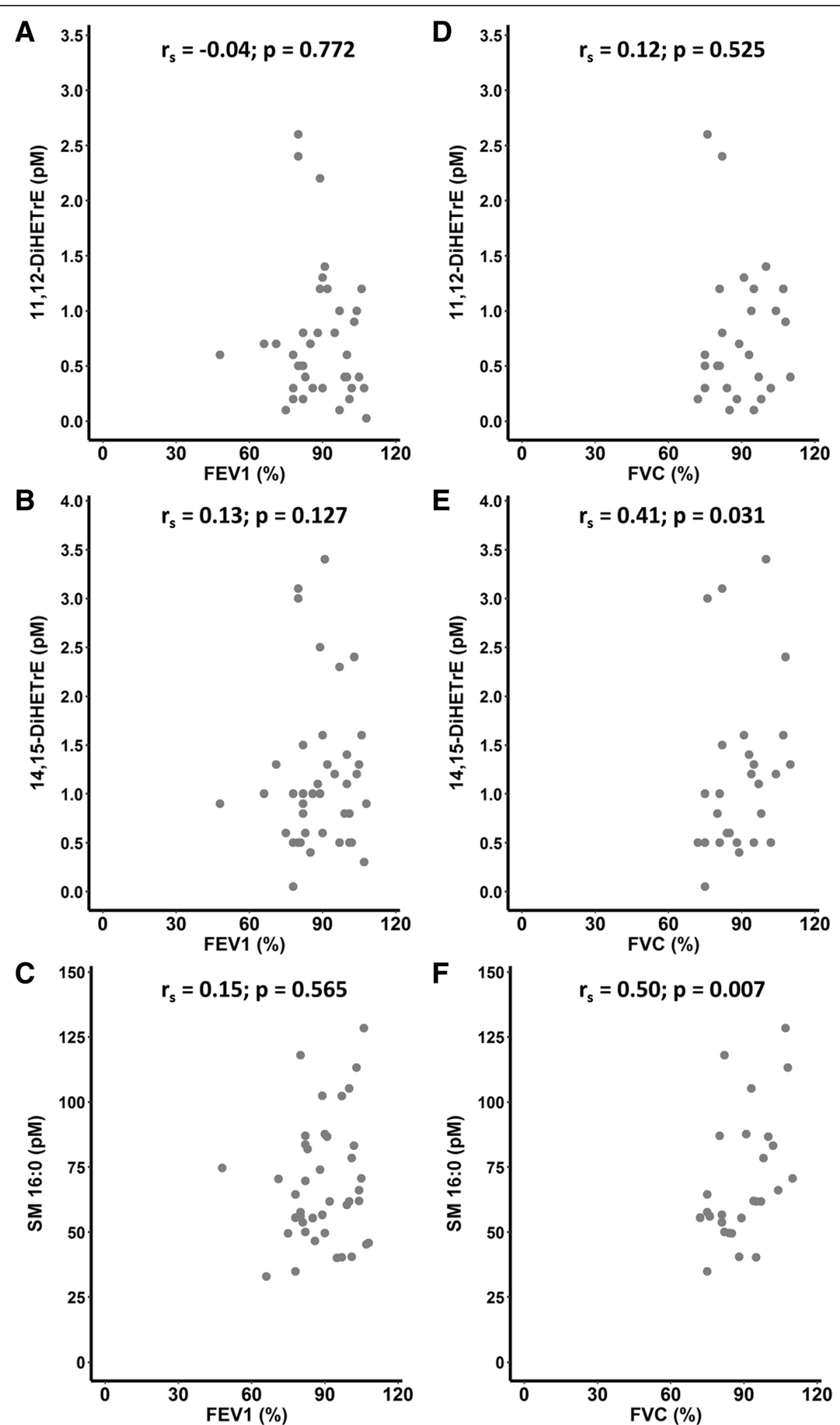

Fig. 4 Associations of lipids differentially expressed in patients with sarcoidosis $(q<0.05)$ and pulmonary function. $(\mathbf{a}-\mathbf{c})$ Spearman's correlation with (a-c) FEV1 (\%) and (d-f) FVC (\%). $r_{s}=$ Spearman's rho. DiHETrE = Dihydroxyeicosatrienoic acid; SM = Sphingomyelin. See Figs. 1 and 2 for compound structures 
stressing the potential role of this pathway in the inflammatory process in lung pathology and highlighting the targeting of this enzyme for disease treatment. It is of particular interest that these sEH products also correlated with radiological stage, further strengthening their association with more advanced disease. These findings suggest that the sEH pathway should be further investigated in sarcoidosis, both from the standpoint of potentially being involved in disease etiology and representing a novel therapeutic target for disease control.

\section{Additional files}

Additional file 1: Data S1. Summary of data used in the analysis. (XLSX $31 \mathrm{~kb}$ )

Additional file 2: Table S2. Nomenclature for the compounds screened in the samples. Compounds reproducibly detected in samples and submitted to data analysis are highlighted in bold. (DOCX $196 \mathrm{~kb}$ )

Additional file 3: Table S3. Internal standards for each of the three LC-MS/MS lipid mediator platforms. (PDF 78 kb)

Additional file 4: Appendix S1. Detailed procedures employed for the extraction of eicosanoids, endocannabinoids and sphingolipids. (PDF $11 \mathrm{~kb}$ )

Additional file 5: Appendix S2. Chromatographic and general MS conditions employed in the detection of eicosanoids, sphingolipids and endocannabinoids. (PDF 97 kb)

Additional file 6: Table S4. Results for the comparisons of lipid mediator levels between healthy $(n=16)$ and sarcoidosis $(n=41)$ groups. Values have been normalized to the median value of the healthy group. Compounds with $p<0.05$ are highlighted in bold. (PDF $71 \mathrm{~kb}$ )

Additional file 7: Table S5. Spearman's correlations between lipid mediators and age for healthy controls and patients with sarcoidosis. Compounds with $p<0.05$ are highlighted in bold. (PDF $39 \mathrm{~kb}$ )

Additional file 8: Table S6. Results of the Cochran-Armitage test with radiography stage in sphingolipids significantly differing $(p<0.05)$ between patients and controls. Direction of the null hypothesis is indicated for each compound. Compounds with $p<0.05$ are highlighted in bold. (PDF $79 \mathrm{~kb}$ )

\section{Abbreviations}

APCs: Antigen presenting cells; BAL: Bronchoalveolar lavage; BHL: Bilateral hilar lymphadenopathy; DiHETrE: Dihydroxyeicosatrienoic acid;

EpOME: Epoxyoctadecenoic acid; FC: Fold change; GalCer: Galactosylceramide; GluCer: Flucosylceramide; HexCer: Hexosylceramide; HLA: Human leukocyte antigen; HODE: Hydroxyoctadecadienoic acid; LC-MS/MS: Liquid chromatography tandem mass spectrometry; LLOQ: Lowest limit of quantification; sEH: Soluble epoxyde hydrolase; SM: Sphingomyelin; SRM: Selected reaction monitoring

\section{Acknowledgements}

The authors would like to acknowledge Stuart Snowden for assistance in extracting the samples and Johan Kolmert for aid in analyzing the samples. The authors thank research nurses Heléne Blomqvist, Margitha Dahl and Gunnel de Forest as well as biomedical analysts Benita Dahlberg and Benita Engvall for skillful assistance with sample collections and preparations.

\section{Funding}

This work was supported by the Swedish Heart Lung Foundation (HLF 20170734, 20170603 and 20160300), the Swedish Research Council (201602798, 2016-01209), The Stockholm County Council, the Swedish Association for Chest Physicians, and the ChAMP (Centre for Allergy Research Highlights Asthma Markers of Phenotype) consortium which is funded by the Swedish Foundation for Strategic Research, the Karolinska Institutet, AstraZeneca \& Science for Life Laboratory Joint Research Collaboration, and the Vårdal Foundation.

\section{Availability of data and materials}

All data generated or analyzed during this study are included as supplementary information files.

\section{Authors' contributions}

$\AA \mathrm{MW}, \mathrm{JG}$ and CEW contributed to the study conception and design. MODS and $A C$ acquired the LC-MS data. AC and MY performed the statistical analyses. MODS, AC, SED, AMW, AE, JG and CEW conceived and wrote the manuscript. All authors read and approved the final manuscript.

\section{Ethics approval and consent to participate}

This study was approved by the Regional Ethical Review Board in Stockholm. Written informed consent was obtained from all subjects.

\section{Consent for publication}

Not applicable.

\section{Competing interests}

The authors declare that they have no competing interests.

\section{Publisher's Note}

Springer Nature remains neutral with regard to jurisdictional claims in published maps and institutional affiliations.

\section{Author details}

${ }^{1}$ Division of Physiological Chemistry II, Department of Medical Biochemistry \& Biophysics, Karolinska Institutet, 17177 Stockholm, Sweden. ${ }^{2}$ Experimental Asthma \& Allergy Research, Institute of Environmental Medicine, Karolinska Institutet, Stockholm, Sweden. ${ }^{3}$ Respiratory Medicine Unit, Department of Medicine and Center for Molecular Medicine (CMM), Karolinska Hospital and Karolinska Institutet, Stockholm, Sweden.

Received: 24 July 2018 Accepted: 14 November 2018 Published online: 03 December 2018

\section{References}

1. Hunninghake GW, Costabel U, Ando M, Baughman R, Cordier JF, du Bois R, Eklund A, Kitaichi M, Lynch J, Rizzato G, et al: Statement on sarcoidosis. Am J Respir Crit Care Med 1999; 160:736-755.

2. Mihailovic-Vucinic V, Jovanovic D. Pulmonary sarcoidosis. Clin Chest Med. 2008;29:459-73.

3. Arkema EV, Grunewald J, Kullberg S, Eklund A, Askling J. Sarcoidosis incidence and prevalence: a nationwide register-based assessment in Sweden. Eur Respir J. 2016.

4. Newman LS, Rose CS, Maier LA. Medical progress - Sarcoidosis. N Engl J Med. 1997:336:1224-34.

5. Muller-Quernheim J. Sarcoidosis: clinical manifestations, staging and therapy (part II). Respir Med. 1998;92:140-9.

6. Valeyre D, Prasse A, Nunes H, Uzunhan Y, Brillet P-Y, Müller-Quernheim J. Sarcoidosis. Lancet. 2014;383:1155-67.

7. Baughman RP, Culver DA, Judson MA. A concise review of pulmonary sarcoidosis. Am J Respir Crit Care Med. 2011;183:573-81.

8. Moller DR, Chen ES. Genetic basis of remitting sarcoidosis - triumph of the trimolecular complex? Am J Respir Cell Mol Biol. 2002;27:391-5.

9. Uderhardt S, Kronke G. 12/15-lipoxygenase during the regulation of inflammation, immunity, and self-tolerance. Journal of Molecular MedicineJmm. 2012;90:1247-56.

10. Grunewald J. Genetics of sarcoidosis. Curr Opin Pulm Med. 2008;14:434-9.

11. Grunewald J. Review: role of genetics in susceptibility and outcome of sarcoidosis. Seminars in Respiratory and Critical Care Medicine. 2010;31: 380-9.

12. Berlin M, FogdellHahn A, Olerup O, Eklund A, Grunewald J. HLA-DR predicts the prognosis in Scandinavian patients with pulmonary sarcoidosis. Am J Respir Crit Care Med. 1997;156:1601-5.

13. Vorselaars ADM, van Moorsel CHM, Zanen P, Ruven HJT, Claessen AME, van Velzen-Blad H, Grutters JC. ACE and sIL-2R correlate with lung function improvement in sarcoidosis during methotrexate therapy. Respir Med. 2015; 109:279-85.

14. Bargagli E, Bennett D, Maggiorelli C, Di Sipio P, Margollicci M, Bianchi N, Rottoli P. Human Chitotriosidase: a sensitive biomarker of sarcoidosis. J Clin Immunol. 2013;33:264-70. 
15. Grutters JC, Fellrath JM, Mulder $L$, Janssen $R$, van den Bosch JMM, van Velzen-Blad H: Serum soluble interleukin-2 receptor measurement in patients with sarcoidosis - a clinical evaluation. Chest 2003; 124:186-195.

16. Bargagli E, Mazzi A, Rottoli P. Markers of inflammation in sarcoidosis: blood, urine, BAL, sputum, and exhaled gas. Clin Chest Med. 2008;29:445.

17. Balgoma D, Checa A, Sar DG, Snowden S, Wheelock CE. Quantitative metabolic profiling of lipid mediators. Mol Nutr Food Res. 2013;57:1359-77.

18. Lemanske RF, Busse WW. Asthma: clinical expression and molecular mechanisms. J Allergy Clin Immunol. 2010;125:S95-S102.

19. Nauta AJ, Engels F, Knippels LM, Garssen J, Nijkamp FP, Redegeld FA. Mechanisms of allergy and asthma. Eur J Pharmacol. 2008;585:354-60.

20. Chung KF, Adcock IM. Multifaceted mechanisms in COPD: inflammation, immunity, and tissue repair and destruction. Eur Respir J. 2008;31:1334-56.

21. Yang J, Eiserich JP, Cross CE, Morrissey BM, Hammock BD. Metabolomic profiling of regulatory lipid mediators in sputum from adult cystic fibrosis patients. Free Radic Biol Med. 2012;53:160-71.

22. Balgoma D, Yang M, Sjodin M, Snowden S, Karimi R, Levanen B, Merikallio H, Kaarteenaho R, Palmberg L, Larsson K, et al. Linoleic acid-derived lipid mediators increase in a female-dominated subphenotype of COPD. Eur Respir J. 2016;47:1645-56.

23. Olsen HH, Grunewald J, Tornling G, Skold CM, Eklund A. Bronchoalveolar lavage results are independent of season, age, gender and collection site. PLoS One. 2012;7:e43644

24. Hunninghake GW, Costabel U, Ando M, Baughman R, Cordier JF, du Bois R, Eklund A, Kitaichi M, Lynch J, Rizzato G, et al: ATS/ERS/WASOG statement on sarcoidosis. Sarcoidosis Vasculitis and Diffuse Lung Diseases 1999; 16:149-173.

25. Checa A, Khademi M, Sar DG, Haeggstrom JZ, Lundberg JO, Piehl F, Olsson T, Wheelock CE. Hexosylceramides as intrathecal markers of worsening disability in multiple sclerosis. Mult Scler. 2015;21:1271-9.

26. Checa A, Holm T, Sjodin MO, Reinke SN, Alm J, Scheynius A, Wheelock CE. Lipid mediator profile in vernix caseosa reflects skin barrier development. Sci Rep. 2015:5:15740.

27. Storey JD. A direct approach to false discovery rates. Journal of the Royal Statistical Society Series B-Statistical Methodology. 2002;64:479-98.

28. Spector AA. Arachidonic acid cytochrome P450 epoxygenase pathway. J Lipid Res. 2009;50:S52-6.

29. Imig JD. Epoxides and soluble epoxide hydrolase in cardiovascular physiology. Physiol Rev. 2012;92:101-30.

30. Morisseau C, Hammock BD: Impact of soluble epoxide hydrolase and Epoxyeicosanoids on human health. In Annual Review of Pharmacology and Toxicology, Vol 53, 2013. Edited by Insel PA: 37-58 Annu Rev Pharmacol Toxicol.

31. Conrad DJ. The arachidonate 12/15 lipoxygenases - a review of tissue expression and biologic function. Clin Rev Allergy Immunol. 1999;17:71-89.

32. Smith KR, Pinkerton KE, Watanabe T, Pedersen TL, Ma SJ, Hammock BD. Attenuation of tobacco smoke-induced lung inflammation by treatment with a soluble epoxide hydrolase inhibitor. Proc Natl Acad Sci U S A. 2005; 102:2186-91.

33. Ono E, Dutile S, Kazani S, Wechsler ME, Yang J, Hammock BD, Douda DN, Tabet $Y$, Khaddaj-Mallat $R$, Sirois $M$, et al. Lipoxin generation is related to soluble epoxide hydrolase activity in severe asthma. Am J Respir Crit Care Med. 2014;190:886-97.

34. Gunther A, Schmidt R, Nix F, Yabut-Perez M, Guth C, Rosseau S, Siebert C, Grimminger F, Morr H, Velcovsky HG, Seeger W. Surfactant abnormalities in idiopathic pulmonary fibrosis, hypersensitivity pneumonitis and sarcoidosis. Eur Respir J. 1999;14:565-73.

35. Merrill AH. Sphingolipid and glycosphingolipid metabolic pathways in the era of Sphingolipidomics. Chem Rev. 2011;111:6387-422.

36. Hartlova A, Cerveny L, Hubalek M, Krocova Z, Stulik J. Membrane rafts: a potential gateway for bacterial entry into host cells. Microbiol Immunol. 2010:54:237-45.

37. Cutler RG, Kelly J, Storie K, Pedersen WA, Tammara A, Hatanpaa K, Troncoso JC, Mattson MP. Involvement of oxidative stress-induced abnormalities in ceramide and cholesterol metabolism in brain aging and Alzheimer's disease. Proc Natl Acad Sci U S A. 2004;101:2070-5.

38. Tibboel J, Reiss I, de Jongste JC, Post M. Sphingolipids in lung growth and repair. Chest. 2014;145:120-8.

39. Moffatt MF, Kabesch M, Liang LM, Dixon AL, Strachan D, Heath S, Depner M, von Berg A, Bufe A, Rietschel E, et al: Genetic variants regulating ORMDL3 expression contribute to the risk of childhood asthma. Nature 2007; 448:470-U475.
40. Masini E, Giannini L, Nistri S, Cinci L, Mastroianni R, Xu W, Comhair SAA, Li D, Cuzzocrea S, Matuschak GM, Salvemini D. Ceramide: a key signaling molecule in a Guinea pig model of allergic asthmatic response and airway inflammation. J Pharmacol Exp Ther. 2008;324:548-57.

41. Rivera J, Proia RL, Olivera A. The alliance of sphingosine-1-phosphate and its receptors in immunity. Nat Rev Immunol. 2008:8:753-63.

42. Ammit AJ, Hastie AT, Edsall LC, Hoffman RK, Amrani Y, Krymskaya VP, Kane SA, Peters SP, Penn RB, Spiegel S, Panettieri RA. Sphingosine 1-phosphate modulates human airway smooth muscle cell functions that promote inflammation and airway remodeling in asthma. FASEB J. 2001;15:1212-4.

43. Lundström SL, Levänen B, Nording M, Klepczynska-Nyström A, Sköld M, Haeggström JZ, Grunewald J, Svartengren M, Hammock BD, Larsson BM, Eklund A, Wheelock ÅM, Wheelock CE. Asthmatics exhibit altered oxylipin profiles compared to healthy individuals after subway air exposure. PLoS One. 2011:6(8):e23864.

44. Wolak JE, Esther CR Jr, O'Connell TM. Metabolomic analysis of bronchoalveolar lavage fluid from cystic fibrosis patients. Biomarkers. 2009: 14(1):55-60.

\section{Ready to submit your research? Choose BMC and benefit from:}

- fast, convenient online submission

- thorough peer review by experienced researchers in your field

- rapid publication on acceptance

- support for research data, including large and complex data types

- gold Open Access which fosters wider collaboration and increased citations

- maximum visibility for your research: over $100 \mathrm{M}$ website views per year

At BMC, research is always in progress.

Learn more biomedcentral.com/submissions 